\title{
MODELOS BIOCLIMÁTICOS DE VERÃO E INVERNO PARA CIDADE MÉdIA EM CLIMA SUBTROPICAL
}

\author{
GOBO, João Paulo Assis - jpgobo@usp.br \\ Universidade Federal de Rondônia/ UNIR \\ FARIA, Marlon Resende - marlon.faria@usp.br \\ Universidade de São Paulo/ USP \\ GALVANI, Emerson - egalvani@usp.br \\ Universidade de São Paulo/ USP
}

\begin{abstract}
RESUMO: Há uma lacuna existente quanto a estudos direcionados a análise do conforto térmico humano em ambientes urbanos de médio e pequeno porte, onde quase metade da população urbana mundial vive. No Brasil, tais estudos são fundamentais pela expressão que as cidades pequenas e médias tomaram nos últimos anos, além de desempenharem papel importante na dinâmica econômica e espacial do país. Deste modo, o presente estudo propôs a definição de dois modelos empíricos sazonais baseado em entrevistas transversais realizadas na cidade de médio porte de Santa Maria - RS, com base nos dados meteorológicos de temperatura do ar, umidade relativa do ar, velocidade do vento e temperatura radiante média, nos dados subjetivos observados nas entrevistas, e nos dados fisiológicos de Índice de Massa Corporal (I.M.C.) obtidos a partir do peso e da altura dos entrevistados. Para tanto, foram obtidos dados meteorológicos por meio de uma estação meteorológica automática instalada no centro da cidade, onde também foram entrevistadas 1720 pessoas por meio de um questionário adaptado do modelo estabelecido pela norma ISO 10551, e, para a obtenção dos modelos, executouse regressões lineares a partir dos dados separados de verão e inverno. Em seguida foi executada a validação dos modelos propostos por meio da comparação entre modelos já tradicionais na literatura, tais como o PET, SET e PMV, os quais foram calibrados para a área de estudo. Os resultados observados propuseram os novos modelos empíricos para áreas urbanas de médio porte em clima subtropical, o Modelo Subtropical de Verão (MSV) e o Modelo Subtropical de Inverno (MSI), os quais foram validados e apresentaram taxa de acerto superior a $80 \%$, superior às taxas de acertos encontradas para índices tradicionais como o PET, SET e o PMV também testados para área de estudo.
\end{abstract}

PALAVRAS-CHAVE: conforto térmico; clima urbano; Modelo Subtropical de Verão; Modelo Subtropical de Inverno.

\section{BIOCLIMATIC MODELS OF SUMMER AND WINTER FOR AVERAGE CITY IN SUBTROPICAL CLIMATE}

ABSTRACT: There is a gap regarding studies about the analysis of human thermal comfort in medium-sized and small urban environments where almost half the world's urban population lives. In Brazil, such studies are fundamental due to the relevance that small and medium-sized cities have acquired in recent years, besides playing a significant role in the country's economic and spatial dynamics. Thus, this study concerns in to proposes a definition of two seasonal empirical models (summer and winter conditions) based on cross-sectional data collected in the medium-sized city of Santa Maria located in the state of Rio Grande do Sul, Brazil. Air temperature, relative air humidity, wind speed, and temperature radiance were obtained from an automatic meteorological station placed in the city center, and also Body Mass Index (BMI) and subjective thermal sensation collected by a survey team that carried out 1720 interviews. These interviews were conducted by means of a questionnaire adapted from the template established by ISO 10551. In order to build the fittings were done using linear regression from the experimental data. Then, the validation of the proposed models was performed by comparing them with pre-existing, such as PET, SET, and PMV, which were calibrated for 
the study area. The observed results allowed for the proposal, new empirical models for subtropical medium-sized urban areas: the Subtropical Summer Model (MSV) and the Subtropical Winter Model (MSI). These were validated with a hit-rate of over $80 \%$, a more significant percentage than those calculated for the calibrated PET, SET and PMV, also tested in the studied area.

KEYWORDS: thermal comfort; urban climate; Subtropical Summer Model; Subtropical Winter Model.

\section{INTRODUÇÃO}

Pesquisadores exploraram durante décadas maneiras de prever a sensação térmica de pessoas em seus ambiente típicos com base em variáveis pessoais, ambientais e fisiológicas (Giannaros et al., 2018). Como resultado, foram desenvolvidos modelos matemáticos que simulam a resposta térmica dos indivíduos nesses ambientes, impulsionados pelo crescimento exponencial da população residente em áreas urbanas (Giannaros et al., 2018; Golasi et al., 2018; Manu et al., 2016; Ruiz and Correa, 2015).

A população brasileira atingiu 190,8 milhões em 2010 e será de aproximadamente 208,9 milhões em 2018 (Ervatti et al., 2015), sendo que a parcela da população com 80 anos ou mais aumentou em aproximadamente $48 \%$ entre 1997 e 2007. Assim, ao considerarmos o crescimento urbano e os padrões climáticos desses ambientes, cabe destacar a vulnerabilidade da população urbana ao estresse térmico, muitas vezes observado nas grandes cidades, porém agora, também percebido em médias e pequenas cidades (Ervatti et al., 2015).

Segundo a Organização das Nações Unidas (United Nations, 2014), metade da população urbana mundial vivia em centros com menos de 500.000 habitantes, sendo que esta população, tende a diminuir ao longo do tempo. No entanto, até 2030, estas cidades menores continuarão a representar cerca de $45 \%$ das áreas urbanas (United Nations, 2014).

No Brasil, tais estudos são fundamentais pela expressão que as cidades pequenas e médias tomaram nos últimos anos, pois nelas se concentram $66,22 \%$ do total da população (IBGE, 2013), além de desempenharem papel importante na dinâmica econômica e espacial do país. Desde modo, segundo o censo demográfico do (IBGE, 2013), 62.575.769 habitantes (38,88\%) viviam em municípios de pequeno porte, com menos de 100 mil habitantes e 43.992.408 habitantes $(27,34 \%)$ em municípios de médio porte com população entre 100.000 e 500.000 habitantes.

O crescimento do envelhecimento populacional, principalmente em cidade de médio e pequeno porte (Hoof and Hensen, 2006; Iommi and Barbera, 2015; Lai et al., 2014; Larose et al., 2014; Organização Mundial da Saúde, 2015; Schellen et al., 2010; Trezza et al., 2015), bem como o aumento da porcentagem de pessoas obesas em todo o planeta (Okada and Kakehashi, 2014; Vanos et al., 2012; WHO, 2017), fazem parte de um padrão crescente do novo modelo de crescimento populacional urbano.

O modo de vida, no cenário contemporâneo, levou as pessoas a passarem mais tempo em ambientes termoneutros uniformes, sem grandes variações de temperatura, bem como sem a necessidade de enfrentar grandes 
desafios térmicos, aos quais o corpo tem de se adaptar em espaços abertos. Todos estes aspectos individuais, associados aos aspectos ambientais dos centros urbanos, levaram ao desenvolvimento de estudos de conforto térmico e predição térmica para populações em espaços abertos, com a finalidade de averiguar os padrões de conforto e preferência da população durante distintas épocas do ano e zonas climáticas (Farajzadeh et al., 2015; Hong and Lin, 2015; Lai et al., 2014; Lin et al., 2011; Nasir et al., 2012; Ng and Cheng, 2012; Pantavou et al., 2018, 2013; Villadiego and Velay-Dabat, 2014; Yang et al., 2013).

Estes estudos concentraram-se em métodos de modelagem e avaliação na perspectiva termofisiológica, tais como os trabalhos de Gulyás et al., (2006) e Höppe (2002), por exemplo, enquanto outros partem da perspectiva da relação entre os parâmetros climáticos que determinam o nível de conforto térmico de seres humanos ao ar livre (Bröde et al., 2012; Cheng and Ng, 2006; Monteiro et al., 2008; Monteiro and Alucci, 2007; Nasir et al., 2012; Rossi et al., 2013, 2012; Spagnolo and de Dear, 2003; Zambrano et al., 2006).

No entanto, a calibração das faixas de conforto térmico de um determinado índice nem sempre responde todas as questões levantadas acerca do conforto térmico de um determinado local. Assim, o presente estudo propõe a definição de dois modelos empíricos sazonais para cidade média de clima subtropical, baseado nas entrevistas transversais realizadas na cidade de Santa Maria, estado do Rio Grande do Sul, Brasil, com base em variáveis ambientais, subjetivas e individuais.

\section{LOCALIZAÇÃO E CARACTERIZAÇÃO DA ÁREA DE ESTUDO}

Localizado no extremo sul do Brasil, o estado do Rio Grande do Sul está situado entre as coordenadas geográficas: $27004^{\prime} 48^{\prime \prime}$ latitude norte, $53^{\circ} 01^{\prime}$ 53" longitude norte, $33^{\circ} 45^{\prime}$ 06" latitude sul, $53^{\circ}$ 23' 48' longitude sul (Figura 1 ), e conta com uma população estimada de 11.322.895 habitantes (IBGE, 2013). 


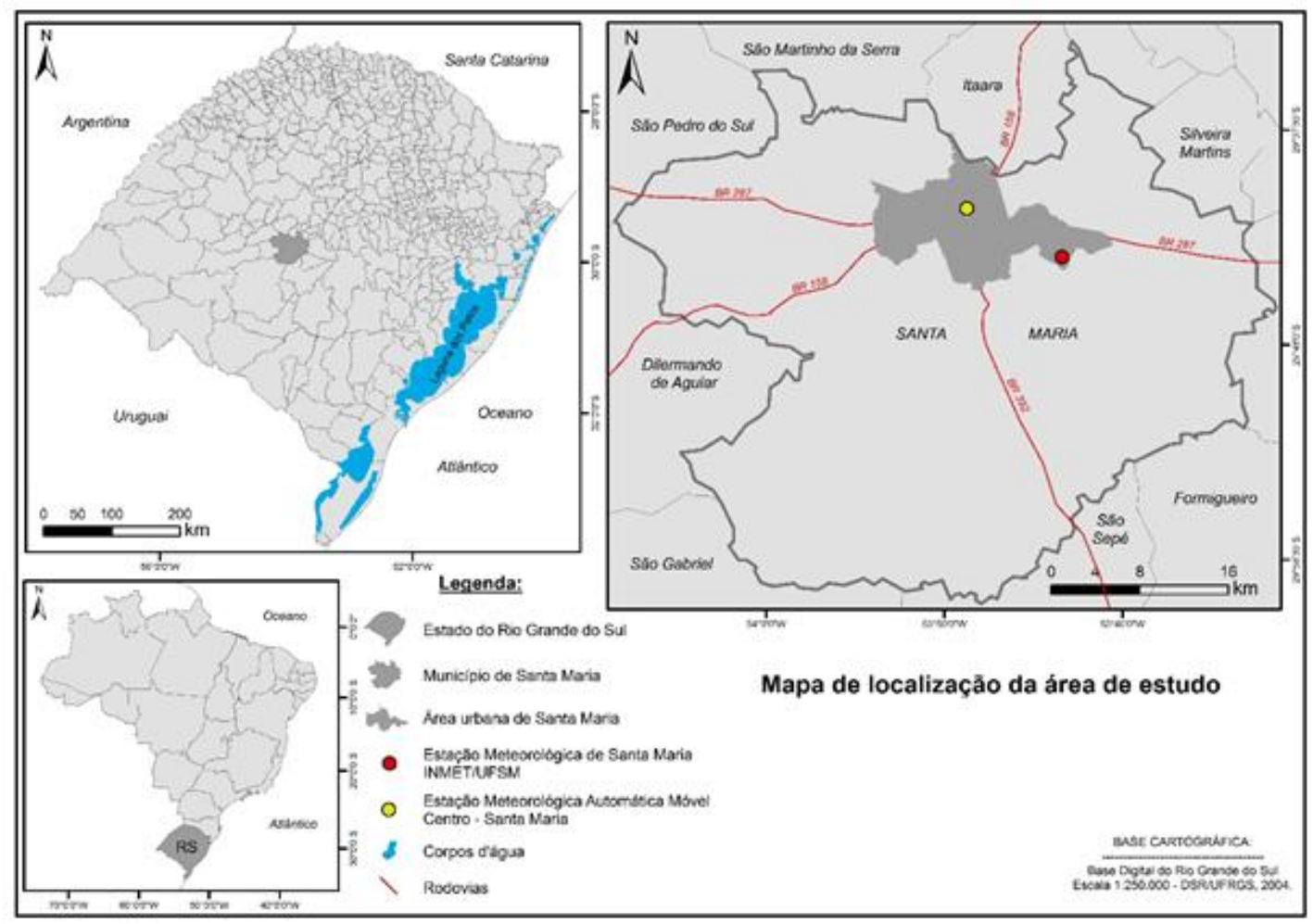

Figura 1 - Mapa de localização da área de estudo.

Já o município de Santa Maria (Figura 1) está situado no centro geográfico do estado, com população estimada em 278.445 habitantes (IBGE, 2013), e é caracterizado, climaticamente segundo Sartori (2016), por um mês mais quente (janeiro) com temperatura média acima de $24^{\circ} \mathrm{C}$ e média das máximas de $32^{\circ} \mathrm{C}$, e um mês mais frio (julho) com temperaturas médias entre $13^{\circ} \mathrm{C}$ e $15^{\circ} \mathrm{C}$, sendo a média das temperaturas mínimas, entre $7^{\circ} \mathrm{C}$ e $10^{\circ} \mathrm{C}$. (Alvares et al., 2013) define Santa Maria dentro do clima Cfa, subtropical com verão quente, porém Dubreuil et al., (2018), aponta para o fato de Santa Maria, além de estar inserida dentro do tipo climático Cfa, também apresenta o tipo climático Csa, clima temperado com verão seco e quente.

\section{CARACTERIZAÇÃo CLIMÁTICA DO PERÍODO DE ANÁLISE}

A coleta de dados de campo foi realizada nos períodos de 5 a 7 de agosto de 2015, 17 a 19 de janeiro de 2016 e de 6 a 8 de julho de 2016, no período das 09h00min às $17 \mathrm{~h} 00 \mathrm{~min}$.

Nos dias do experimento de campo, no período de 5 a 7 de agosto de 2015 houve uma persistente anomalia no sistema de alta pressão (anticiclone posicionado a aproximadamente 30 graus de latitude Sul), atuando por vários dias. Durante os dias de pesquisa de campo de janeiro de 2016, foi possível identificar um padrão compatível com as médias climatológicas normais de Santa Maria para aquele mês que apresentou temperaturas elevadas com máximas acima de $32^{\circ} \mathrm{C}$ (Tabela 1 ). 
Na análise feita durante o inverno, julho de 2016, foram observadas temperaturas superiores a média para Santa Maria, principalmente no primeiro dia de análise. Após esse dia, as temperaturas ficaram dentro da faixa esperada para aquela estação (Tabela 1).

Tabela 1 - Valores médios dos atributos climáticos coletados durante o período de análise.

\begin{tabular}{ccccccccc}
\hline DIAS & $\begin{array}{c}\text { Temperatura Temperatura Temperatura } \\
\text { Média }\left({ }^{\circ} \mathbf{C}\right)\end{array}$ & $\begin{array}{c}\text { Umidade } \\
\text { mínima }\left({ }^{\circ} \mathbf{C}\right)\end{array}$ & $\begin{array}{c}\text { Umidade } \\
\text { máxima }\left({ }^{\circ} \mathbf{C}\right) \\
\text { Relativa } \\
\text { média(\%) }\end{array}$ & $\begin{array}{c}\text { Umidade } \\
\text { mínima } \\
(\%)\end{array}$ & $\begin{array}{c}\text { Relativa } \\
\text { Máxima } \\
(\%)\end{array}$ & $\begin{array}{c}\text { Velocidade Velocidade } \\
\text { do vento } \\
\text { média } \\
(\mathbf{m} / \mathbf{s})\end{array}$ & $\begin{array}{c}\text { do vento } \\
\text { máxima } \\
(\mathbf{m} / \mathbf{s})\end{array}$ \\
\hline $05 / 08 / 2015$ & 22,9 & 18,0 & 25,7 & 73,1 & 65,0 & 88,2 & 0,0 & 1,1 \\
$06 / 08 / 2015$ & 28,4 & 24,4 & 30,7 & 37,6 & 32,0 & 47,2 & 1,4 & 8,8 \\
$07 / 08 / 2015$ & 29,3 & 26,4 & 31,4 & 34,4 & 30,9 & 38,2 & 1,3 & 8,3 \\
\hline $17 / 01 / 2016$ & 30,0 & 21,1 & 35,2 & 52,7 & 23,9 & 86,7 & 0,1 & 2,5 \\
$18 / 01 / 2016$ & 31,5 & 23,6 & 36,6 & 36,3 & 11,4 & 59,8 & 0,1 & 2,3 \\
$19 / 01 / 2016$ & 30,5 & 23,5 & 36,7 & 46,9 & 23,6 & 70,8 & 0,1 & 2,2 \\
\hline $06 / 07 / 2016$ & 10,5 & 8,1 & 12,9 & 79,7 & 70,3 & 89,8 & 0,5 & 3,1 \\
$07 / 07 / 2016$ & 16,0 & 11,4 & 18,4 & 51,2 & 38,5 & 74,3 & 0,2 & 2,3 \\
$08 / 07 / 2016$ & 17,2 & 11,3 & 19,7 & 52,9 & 44,1 & 71,9 & 0,0 & 0,7 \\
\hline
\end{tabular}

\section{MATERIAIS E MÉTODOS}

Os dados meteorológicos foram coletados durante 2015 e 2016 por meio de uma Estação Meteorológica Automática Campbell CR100 (AWS) contendo os seguintes sensores: sensor de precipitação; de radiação global; de temperatura e umidade relativa; de velocidade e direção do vento; e termômetro de globo cinza. A estação foi posicionada em uma área pavimentada no centro de Santa Maria.

Foram entrevistadas apenas pessoas residentes em Santa Maria a mais de um ano, em função da história térmica e memória ambiental dos inqueridos, como foi observado por (Nikolopoulou et al., 2001), totalizando 1720 entrevistas. Também foram limitadas as entrevistas a pessoas com área de corpo coberta entre 0,5 e 1,0 clo, o que corresponde, respectivamente, a um indivíduo de calça jeans e camiseta e um indivíduo de terno (ISO 9920, 2007) e com um padrão de atividade física de $300 \mathrm{~W}$, uma vez que só foram selecionadas pessoas em movimento (caminhando) (ISO, 2004). Quanto ao questionário aplicado, foi utilizado o modelo estabelecido pela norma ISO 10551 (1995) (Figura 2). 


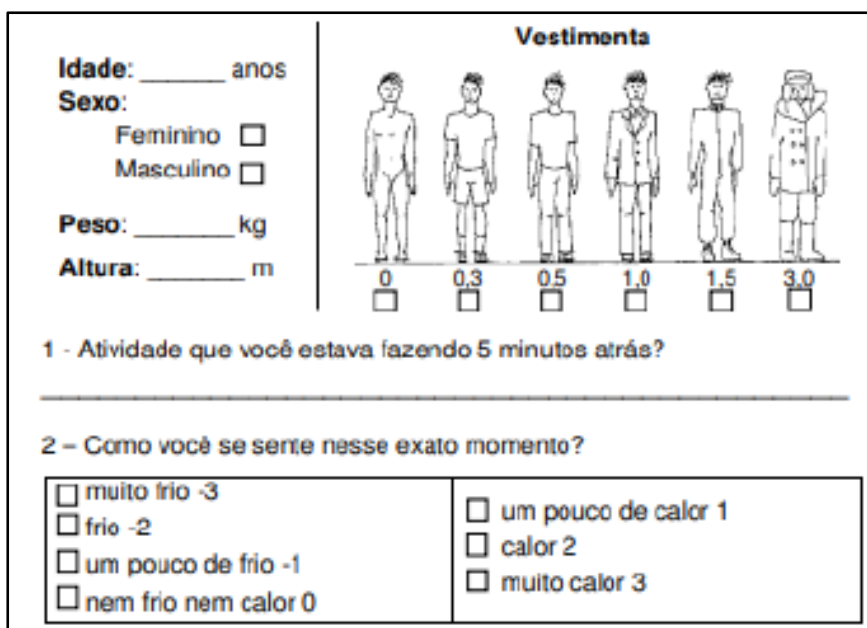

3 - Neste exato momento, com relaçằo ao clima, eu estou:

\begin{tabular}{|l|l|}
\hline $\begin{array}{l}\square \text { confortável 0 } \\
\square \text { um pouco desconfortável 1 }\end{array}$ & $\square$ desconfortável 2 \\
$\square$ muito desconiortável 3 \\
\hline
\end{tabular}

4 - Neste exato momento eu preferia estar sentindo:

\begin{tabular}{|l|l|}
\hline$\square$ muito mais frio -3 & $\square$ um pouco mais de calor 1 \\
$\square$ mais frio -2 & $\square$ mais calcr 2 \\
$\square$ um pouco mais de frio-1 & $\square$ muito mais calor 3 \\
$\square$ sem mudanças 0 &
\end{tabular}

5 - Considerando apenas a sua preferència pessoal, você aceita ou rejeita as condiçōes clmáticas deste ambiente?

\begin{tabular}{|l|l|}
\hline$\square$ aceita & $\square$ Rejeita \\
\hline
\end{tabular}

Figura 2 - Questionário aplicado.

Por meio da correlação das variáveis ambientais (temperatura do ar, umidade relativa do ar e temperatura radiante média - TRM) e variáveis fisiológicas (índice de massa corporal - I.M.C.) observadas em campo com as respostas subjetivas dos entrevistados, um novo modelo empírico foi proposto. Para tanto, foram utilizados apenas os dados referentes aos dias de coleta nos meses de janeiro e julho de 2016, uma vez que os dados de agosto de 2015 foram utilizados posteriormente para a validação dos resultados do modelo.

Para o cálculo da TRM utilizou-se os dados de um termômetro de globo combinado com medições de temperatura do ar e velocidade do vento (Equação 1) (Johansson et al., 2014; Thorsson et al., 2007).

\section{Equação 1}

$$
\operatorname{TRM}=\left[\left(t_{g}+273\right)^{4}+1,1 \cdot \frac{10^{8} \cdot V^{0,6}}{\varepsilon \cdot d^{0,4}} \cdot\left(t_{g}-t_{a}\right)\right]^{0,25}-273
$$


Em que: TRM=temperatura radiante média $\left({ }^{\circ} \mathrm{C}\right) ; \mathrm{tg}=$ temperatura do termômetro de globo $\left({ }^{\circ} \mathrm{C}\right)$; ta $=$ temperatura de bulbo seco do ambiente $\left({ }^{\circ} \mathrm{C}\right)$; $\mathrm{v}=$ velocidade do ar na altura do globo (m/s); $\varepsilon=$ emissividade do globo; $\mathrm{d}=$ diâmetro do globo $(\mathrm{m})$.

Na obtenção dos dados de índice de massa corporal (I.M.C.), considerouse o peso e a altura para classificar o baixo e o excesso de peso, além da obesidade dos adultos, a partir dos parâmetros indicados pela Organização Mundial da Saúde - OMS (World Health Organization, 2006). Este índice é definido como o peso em quilogramas dividido pelo quadrado da altura em metros (kg.m-2). A OMS subdivide o I.M.C. de um indivíduo de acordo com as seguintes classes:

- menor que 18,5 - abaixo do peso;

- entre 18,5 e 24,9 - peso normal;

- entre 25 e 29,9 - sobrepeso;

- igual ou acima de 30 - obesidade.

A presente pesquisa optou por adotar apenas três faixas de classificação do I.M.C. corporal dos indivíduos amostrados, são elas:

- menor que 18,5 - abaixo do peso;

- entre 18,5 e 24,9 - peso normal;

- acima de 25 - acima do peso.

Já para a formulação do modelo foram selecionados, $66 \%$ dos dados com a finalidade de obter o modelo linear pelo método dos mínimos quadrados e logística multivariada, a fim de compará-los. O restante dos dados, $34 \%$ foram utilizados para validação.

A correlação de Pearson de $98,9 \%$ entre os modelos de regressão logística e linear mostrou desnecessária a utilização de dois métodos diferentes, e, portanto, optou-se pelo modelo de regressão linear múltipla descrito pela seguinte expressão matemática:

\section{Equação 2}

$$
Y=\alpha_{1} x_{1}+\alpha_{2} x_{2}+\alpha_{3} x_{3}+\cdots \alpha_{n} x_{n}+c
$$

Em que: n é o número de termos da equação; an são os parâmetros (constantes) obtidos pela regressão; c é a constante que corresponde ao ponto onde a reta ajustada intercepta o eixo y; Y é a variável dependente; xn são as variáveis independentes. foram:

No caso da pesquisa em questão, as variáveis e parâmetros observados

\section{Equação 3}

Índice Empírico $=\alpha_{1}$ Tar $+\alpha_{2} \mathrm{UR}+\alpha_{3} \mathrm{IMC}_{\text {média }}+\alpha_{4} \mathrm{TRM}+\mathrm{C}$ 
Em que: Tar é temperatura do ar $\left({ }^{\circ} \mathrm{C}\right)$; UR é a umidade relativa do ar (\%); I.M.C.média é a média do I.M.C. dos entrevistados; TRM é a temperatura radiante média e $C$ que é a constante numérica.

O que chamamos de Índice Empírico é a média das respostas observadas em campo para um dado valor das variáveis climáticas durante o intervalo de 20 minutos. Considerando que são possíveis apenas valores discretos de -3 a 3 , de acordo com a escala da (Ansi/Ashrae, 2004), a média das respostas foi obtida a partir do cálculo da média de uma distribuição discreta aleatória.

O método de regressão linear múltipla é simples e robusto, desde que verificados os pressupostos de linearidade dos dados, tais como normalidade (obtida pela medida de obliquidade e curtose da distribuição) heterocedasticidade e covariância, os quais, foram, por fim, executados no processo de validação do modelo (Tabachnick and Fidell, 2012).

Para a validação do modelo desenvolvido, foram utilizados $33 \%$ dos dados para a comparação entre este e os modelos já tradicionais na literatura, Physiologically Equivalent Temperature - PET (Höppe, 1999), Standard Effective Temperature - SET* (Pickup and De Dear, 2000) e Predicted Mean Vote - PMV (Fanger, 1970), os quais tiveram suas classes ajustadas por Gobo, Galvani e Wollmann, (2018) para a mesma situação climática em Santa Maria.

\section{RESULTADOS}

Primeiramente, ao buscar-se avaliar uma nova proposta de modelo empírico para duas situações sazonais, verão e inverno, analisou-se as correlações entre as variáveis utilizadas e a preferência térmica dos indivíduos (Índice Empírico), as quais são apresentadas a partir do gráfico da Figura 3. 

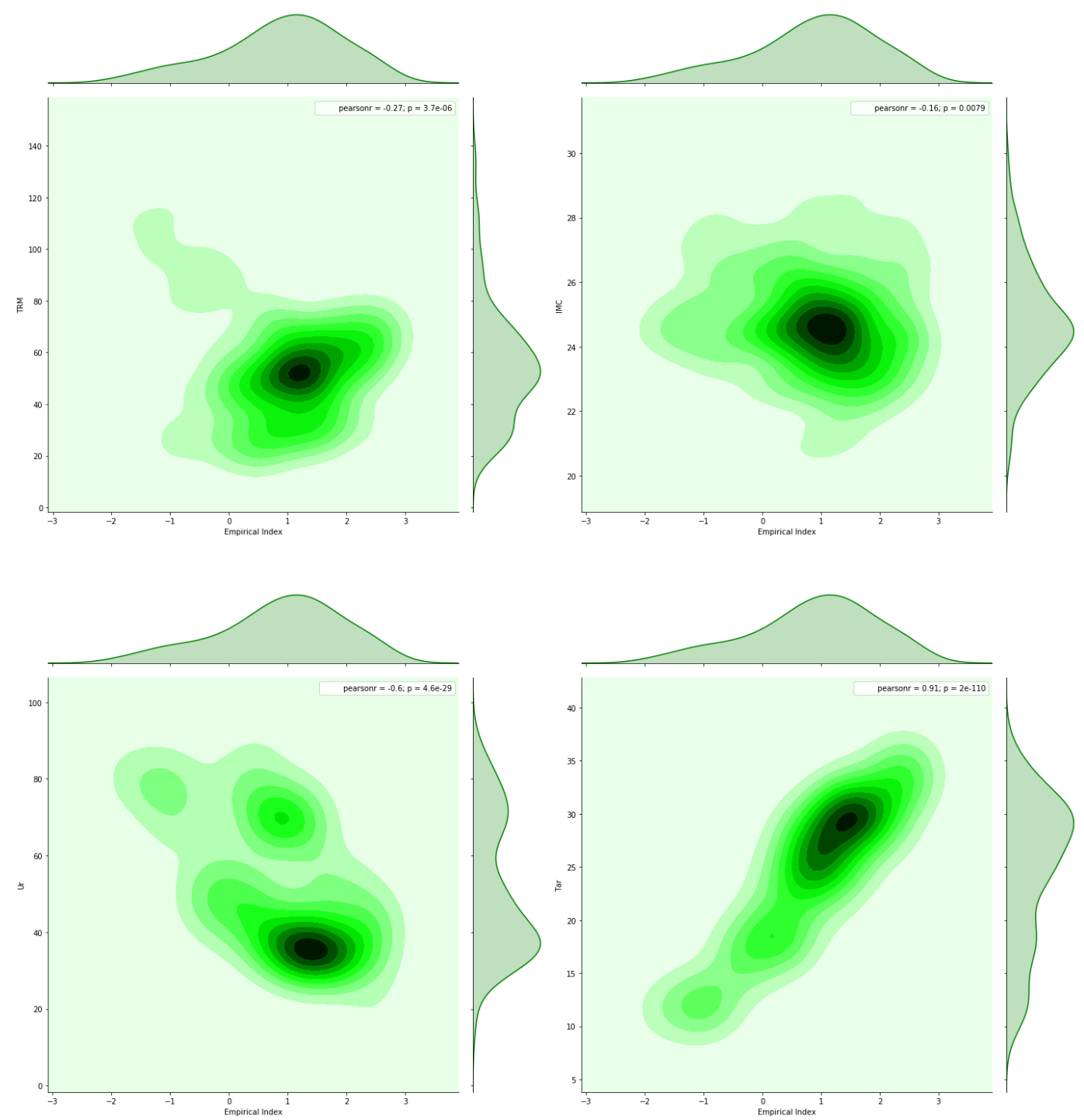

Figura 3 - Correlação média entre as variáveis utilizadas (Tar, UR, TRM e IMC) (janeiro e julho de 2016).

Observa-se uma correlação positiva elevada entre as médias dos votos de preferência térmica e a temperatura do ar (Tar), com valor de R 0,91. A correlação entre o Índice Empírico e a TRM apresentou valor de R negativo baixo a moderado de $-0,27$.

Em relação à umidade relativa do ar, o Índice Empírico mostrou correlação negativa elevada, com $R$ igual a $-0,69$. Já à correlação entre o Índice Empírico e o I.M.C., apresentou correlação negativa fraca com R igual a -0,16.

No que diz respeito à distribuição das médias dos votos de preferência térmica dos indivíduos entrevistados (Índice Empírico), concentrou-se em duas faixas preferenciais de sensação térmica, -1 a 0 e 2 a 3 (escala de 7 pontos (Ansi/Ashrae, 2004)). Bem como a média dos votos de sensação térmica, a 
distribuição média da temperatura do ar apresentou dois pontos de concentração, entre $10^{\circ} \mathrm{C}$ e $20^{\circ} \mathrm{C}$ e acima de $30^{\circ} \mathrm{C}$.

A correlação da temperatura do ar com a umidade relativa apontou um $\mathrm{R}$ de $-0,76$ o que demonstra multicolinearidade entre as variáveis. Em relação ao I.M.C., a temperatura do ar apresenta correlação negativa fraca, com $\mathrm{R}$ igual a 0,256 , e em relação à TRM, a correlação com a temperatura do ar também é negativa e fraca, com $\mathrm{R}$ de $-0,392$.

Já a umidade relativa do ar apresentou correlação positiva, porém fraca com o I.M.C., assim como com a TRM, com R de 0,0588. A distribuição média da umidade relativa revela concentração elevada entre 40 e $50 \%$.

O I.M.C. e a TRM apresentaram correlação negativa fraca, com valor de R igual a -0,0992. A distribuição do I.M.C. ficou em torno de 24 e 25, o que corresponde ao limite entre o I.M.C. considerado normal e o sobrepeso.

Em função da multicolinearidade observada, foi executada uma regressão substituindo a variável sob suspeita (UR) por outra que em tese não seria afetada (Umidade Absoluta- UA), porém, os resultados da regressão com a UA não demonstraram melhora estatística considerável, sendo que o nível de significância da UA ( $p$-value 0,72 ) é inferior à UR ( $p$-value 0,27 ).

Assim, calculou-se o Fator de Inflação da Variância (VIF) para as variáveis previamente selecionadas. Como afirma Marquardt (1970), aquelas variáveis caracterizadas por valores de VIF maiores que 10 devem ser excluídas, no entanto, as variáveis colineares não acrescentam qualquer valor relevante ao modelo, não sendo verificados valores acima do limiar estabelecido por Marquardt (1970) (Tabela 2), logo, optou-se, por manter a umidade relativa na construção do modelo.

Tabela 2 - Resultado do Fator de Inflação da Variância (VIF) para cada variável utilizada na execução do modelo.

\begin{tabular}{cc}
\hline Variáveis & Fator de Inflação da Variância (VIF) \\
\hline Tar & 2.476 \\
UR & 2.263 \\
I.M.C & 1.071 \\
TRM & 1.174 \\
\hline
\end{tabular}

A seguir, na Tabela 3 apresenta-se os resultados das regressões lineares realizadas para os valores médios alcançados para as 72 situações levantadas durante o verão. 
Tabela 3 - Análise estatística da constante, das quatro variáveis independentes e da variância para a regressão realizada para o verão (janeiro de 2016).

\begin{tabular}{lcccc}
\hline & Coeficiente & Erro Padrão & Valor Estatístico t & Nível de significância p \\
\hline Constante & $-4,103611$ & 1,912679 & $-2,145$ & 0,0355 \\
Tar & 0,141870 & 0,050093 & 2,832 & 0,0061 \\
UR & 0,002138 & 0,009280 & 0,230 & 0,8185 \\
I.M.C. & 0,060335 & 0,029102 & 2,073 & 0,0420 \\
TRM & 0,001708 & 0,006119 & 0,279 & 0,7810 \\
\hline Erro padrão residual & & & 0,3882 \\
R - Múltiplo quadrado & & & 0,6616 \\
R - Quadrado ajustado & & 0,6414 \\
F - Estatístico & & 32,75 \\
Nível de Significância - $\mathbf{p}$ & & 3,987 \\
\hline
\end{tabular}

O valor estatístico t testa a hipótese nula de que o coeficiente da variável independente seja igual a 0 , ou seja, que ela não contribui para a predição da variável dependente. $O$ valor de t é a razão entre o coeficiente de regressão e o seu erro padrão. Os valores de $\mathrm{p}$ apresentados referem-se à predição da variável dependente por combinação linear das variáveis independentes.

Verificam-se resultados próximos, com a soma dos quadrados totais praticamente idêntica, em ambos os casos. Contudo, por meio do teste estatístico $F$, que considera a razão entre os quadrados médios, o valor não é tido como elevado, no entanto, indica que as variáveis em conjunto contribuem para a predição da variável independente.

Assim, com base nos resultados encontrados, chegou-se a uma equação que considera as quatro variáveis anteriormente apresentadas (Tar, UR, TRM e I.M.C.). Porém, ainda que se assuma o efeito da umidade na sensação de conforto, sabe-se que este é distinto em diversas regiões, devido à aclimatação das pessoas, como justificado por Monteiro (2008). Então, tais variáveis foram correlacionadas para as 72 situações levantadas e o valor médio de percepção de sensação térmica verificado em cada uma delas (tendo como base os resultados dos 432 questionários aplicados em janeiro de 2016), tem-se a proposição da equação seguinte:

\section{Equação 4}

$$
\mathrm{MSV}=0,1419 \times \operatorname{Tar}+0,0021 \times U R+0,0603 \times I M C_{\text {médio }}+0,0017 \times T R M-4,1036
$$

Em que: Tar $=$ temperatura do ar $\left({ }^{\circ} \mathrm{C}\right) ;$ UR $=$ umidade relativa do ar (\%); I.M.C.média = índice de massa corporal médio dos entrevistados; TRM = temperatura radiante média $\left({ }^{\circ} \mathrm{C}\right) ; \mathrm{MSV}=$ Modelo Subtropical de Verão.

Após a proposição do Modelo Subtropical de Verão (MSV), buscou-se avaliar uma nova proposta de índice empírico para a situação de inverno, a partir dos dados coletados em julho de 2016.

A Tabela 4 expõe os resultados das regressões lineares realizadas para os valores médios alcançados para as 72 situações levantadas durante 0 inverno. 
Tabela 4 - Análise estatística da constante, das quatro variáveis independentes e da variância para a regressão realizada para o inverno (julho de 2016).

\begin{tabular}{lcccc}
\hline & Coeficiente & Erro Padrão & Valor Estatístico t & Nível de Significância \\
\hline Constante & $-2,6442001$ & 1,6867833 & $-1,568$ & 0,12169 \\
Tar & 0,1438950 & 0,0522399 & 2,755 & 0,00756 \\
UR & 0,0109601 & 0,0102163 & $-1,073$ & 0,28721 \\
I.M.C. & 0,0259737 & 0,0276817 & 0,938 & 0,35146 \\
TRM & 0,0004312 & 0,0019910 & $-0,217$ & 0,82921 \\
\hline Erro padrão residual & & & 0,3711 \\
R - Múltiplo Quadrado & & & 0,729 \\
R - Quadrado Ajustado & & & 0,7128 \\
F - Estatístico & & & 45,06 \\
Nível de Significância - p & & & $<2,2$ \\
\hline
\end{tabular}

A soma dos quadrados totais, novamente, apresentou-se praticamente idêntica em ambos os casos, porém com valores mais elevados que os verificados para o verão. Por meio do teste estatístico $F$, que considera a razão entre os quadrados médios, o valor novamente não é considerado elevado, no entanto indica que as variáveis em conjunto contribuem para a predição da variável independente.

Definiu-se, então, a equação que considera as quatro variáveis anteriormente apresentadas correlacionadas para as 72 situações levantadas e 0 valor médio de percepção de sensação térmica verificado em cada uma delas (tendo como base os resultados dos 432 questionários aplicados em julho de 2016):

\section{Equação 5}

$$
M S I=0,1439 \times \text { Tar }-0,0110 \times U R+0,0260 \times I M C_{\text {médio }}-0,0004 \times T R M-2,6442
$$

Em que: Tar $=$ temperatura do $\operatorname{ar}\left({ }^{\circ} \mathrm{C}\right) ;$ UR $=$ umidade relativa do ar $(\%) ;$ I.M.C.média = índice de massa corporal médio dos entrevistados; TRM = temperatura radiante média $\left({ }^{\circ} \mathrm{C}\right) ; \mathrm{MSI}=$ Modelo Subtropical de Inverno.

Ambos os modelos, MSI e MSV, podem ser sucintamente definido como uma escala de sensação térmica baseada no voto médio dos indivíduos entrevistados, por meio da escala de 7 pontos (Tabela 5).

Tabela 5 - Faixas interpretativas para os modelos MSV e MSI.

\begin{tabular}{cl}
\hline$<=-3$ & Muito frio \\
$-2,9$ a -2 & Frio \\
$-1,9$ a -1 & Um pouco de frio \\
$-0,9$ a 0,9 & Nem frio nem calor \\
1 a 1,9 & Um pouco de calor \\
2 a 2,9 & Calor \\
$>=3$ & Muito calor \\
\hline
\end{tabular}

Cabe ressaltar que a equação foi obtida a partir de dados provenientes de uma situação típica de inverno e verão para a cidade de Santa Maria - RS e a utilização em outras situações ou ambientes depende da verificação de correlação de resultados com dados observados. 


\section{VALIDAÇÃO DOS MODELOS}

Após a execução e o desenvolvimento dos modelos anteriormente apresentado, cabe abordar o processo de validação dos mesmos. Para tanto, foram utilizados dados obtidos por meio do primeiro trabalho de campo realizado em agosto de 2015.

A correlação entre o MSV e o MSI com as respostas de sensação térmica da população observadas em campo no período de agosto de 2015, estão descritas na Tabela 6.

Dentre os índices de conforto térmico mais utilizados na literatura, 4 (PET, PMV, Universal Thermal Climate Index - UTCI, SET*) são amplamente utilizados em estudos de percepção térmica ao ar livre (Potchter et al., 2018). Deste modo, realizou-se um teste de correlação entre as respostas de preferência térmica dos entrevistados (Índice Empírico) no período utilizado para a validação dos modelos MSV e MSI (Agosto de 2015) e os modelos PET (Höppe, 1999), SET* e PMV calibrados por Gobo, Galvani e Wollmann (2018) para a Santa Maria. Os resultados também estão descritos na tabela 6 .

Tabela 6 - Correlação entre os modelos MSV, MSI e os modelos PET, SET* e PMV em relação a preferência térmica dos entrevistados (Índice Empírico).

\begin{tabular}{c}
\hline $\begin{array}{c}\text { Correlação entre os modelos MSV e MSI e voto médio dos } \\
\text { indivíduos entrevistados no período de Agosto de } 2015\end{array}$ \\
MSV $=0,8305$ \\
$M S I=0,8264$ \\
\hline $\begin{array}{c}\text { Correlação entre os modelos PET, SET* e PMV calibrados por } \\
\text { Gobo, Galvani e Wollmann (2018) e voto médio dos indivíduos } \\
\text { entrevistados no período de Agosto de 2015. }\end{array}$ \\
\hline $\mathrm{PET}^{\text {calibrado }}=0,2352$ \\
$\mathrm{SET}^{*}$ calibrado $=0,1478$ \\
$\mathrm{PMV}$ Calibrado $=0,38054$
\end{tabular}

Observou-se uma eficiência muito baixa dos modelos PET, SET* e PMV, mesmo calibrados para o ambiente em questão, quando comparados aos modelos propostos. No entanto, é importante destacar que as correlações feitas entre os modelos PET, SET*, PMV e o Índice Empírico foram apenas para o período utilizado na validação dos modelos MSV e MSI, de agosto de 2015, diferentemente do período utilizado por Gobo, Galvani e Wollmann (2018) na calibração dos referidos modelos, onde utilizou-se toda a série de agosto de 2015, janeiro e julho de 2016.

Segundo Farajzadeh et al. (2015) e Potchter et al. (2018), os índices baseados em fórmulas relativamente simples apresentaram baixa correlação com índices termofisiológicos. A provável razão para essa falta de conformidade, segundo Farajzadeh et al. (2015), é a falta de fator de radiação nas equações simples.

A baixa eficiência dos índices PET, SET* e PMV para o período analisado pode se dar, em partes, pelo amplo tamanho da faixa de conforto destes índices calibrada por Gobo, Galvani e Wollmann (2018) para a área de estudo. 


\section{CONSIDERAÇÕES FINAIS}

A proposição de modelos empíricos sazonais de conforto térmico baseado em pesquisa de campo transversal é uma contribuição inédita para a bioclimatologia humana em cidades de médio porte da zona subtropical brasileira. O Modelo Subtropical de verão (MSV) e o Modelo Subtropical de Inverno (MSI), propostos nesta pesquisa, apresentaram F-estatístico e R2 ajustado elevados, não revelando nenhum comportamento patológico que indicasse inapropriação dos modelos escolhidos.

A validação dos modelos confirmou o alto desempenho destes, bem como sua maior eficiência na área de estudo quando comparados aos demais índices comumente utilizados em estudos para espaços abertos na literatura.

Assim, como qualquer modelo estatístico, o MSV e o MSI possuem limitações e são válidos para ambientes característicos de sua formulação, a cidade de médio porte de Santa Maria-RS localizada sob influência de clima subtropical, sendo que para a sua utilização em um espectro ambiental mais amplo, recomenda-se a sua validação e, quando necessária, sua calibração.

Mais estudos são necessários acerca da eficácia dos modelos introduzidos nesta pesquisa, há necessidade de uma abordagem maior, baseada em análise diária mais abrangente e em uma maior variedade de condições climáticas a serem testadas, especialmente considerando aspectos mais detalhados das características e preferências dos indivíduos entrevistados, como a distribuição demográfica local, etnia, distribuição socioeconômica e histórico térmico, bem como o estilo de vida e a frequência do uso do ar condicionado, especialmente em locais com estações climáticas bem definidas, como a área de estudo da presente pesquisa.

\section{AGRADECIMENTOS}

Os autores desta pesquisa agradecem ao Conselho Nacional de Desenvolvimento Científico e Tecnológico (CNPq), aos alunos do LaCAS Laboratório de Climatologia Ambiental e Subtropical da Universidade Federal de Santa Maria (UFSM), aos colegas Thiago Silveira, Daniela Onça, Celso Borzani e Rogério Alves, todos os quais auxiliaram no desenvolvimento do trabalho de campo desta pesquisa e, em especial, aos moradores da cidade de Santa Maria, Rio Grande do Sul, Brasil.

\section{REFERÊNCIAS}

Alvares, C.A., Stape, J.L., Sentelhas, P.C., De Moraes Gonçalves, J.L., Sparovek, G., 2013. Köppen's climate classification map for Brazil. Meteorol. Zeitschrift 22, 711-728. https://doi.org/10.1127/0941-2948/2013/0507

Ansi/Ashrae, 2004. ANSI/ASHRAE 55:2004 Thermal Environmental Conditions for Human Occupancy. Ashrae. https://doi.org/10.1007/s11926-011-0203-9

Bröde, P., Fiala, D., Blazejczyk, K., Holmér, I., Jendritzky, G., Kampmann, B., Tinz, B., Havenith, G., 2012. Deriving the operational procedure for the Universal Thermal Climate Index (UTCI). Int. J. Biometeorol. https://doi.org/10.1007/s00484-011-0454-1 
Cheng, V., Ng, E., 2006. Thermal Comfort in Urban Open Spaces for Hong Kong. Archit. Sci. Rev. https://doi.org/10.3763/asre.2006.4932

Ervatti, L.R., Borges, G.M., Jardim, A. de P., 2015. Mudança Demográfica no Brasil no início do Século XXI. Subsidios para as Projeções da População, IBGE, Instituto Brasileiro de Geografia e Estatística. https://doi.org/ISSN 0101-4234

Fanger, P.O., 1970. Thermal comfort, analysis and application in environmental engineering. Copenhagen Danish Tech. Press.

Farajzadeh, H., Saligheh, M., Alijani, B., Matzarakis, A., 2015. Comparison of selected thermal indices in the northwest of Iran. Nat. Environ. Chang. 1, 1-20.

Giannaros, T.M., Lagouvardos, K., Kotroni, V., Matzarakis, A., 2018. Operational forecasting of human-biometeorological conditions. Int. J. Biometeorol. 62, 1339-1343. https://doi.org/10.1007/s00484-018-1525-3

Gobo, J. P. A.; Galvani, E.; Wollmann, C.A., 2018. Subjective Human Perception of Open Urban Spaces in the Brazilian Subtropical Climate: A First Approach. climate 6, 1-12. https://doi.org/10.3390/cli6020024

Golasi, I., Salata, F., de Lieto Vollaro, E., Coppi, M., 2018. Complying with the demand of standardization in outdoor thermal comfort: a first approach to the Global Outdoor Comfort Index (GOCI). Build. Environ. 130, 104-119. https://doi.org/10.1016/j.buildenv.2017.12.021

Gulyás, Á., Unger, J., Matzarakis, A., 2006. Assessment of the microclimatic and human comfort conditions in a complex urban environment: Modelling and measurements. Build. Environ. https://doi.org/10.1016/j.buildenv.2005.07.001

Hong, B., Lin, B., 2015. Numerical studies of the outdoor wind environment and thermal comfort at pedestrian level in housing blocks with different building layout patterns and trees arrangement. Renew. Energy 73, 18-27. https://doi.org/10.1016/j.renene.2014.05.060

Hoof, J. Van, Hensen, J.L.M., 2006. Thermal comfort and older adults. Gerontechnology 4. https://doi.org/10.4017/gt.2006.04.04.006.00

Höppe, P., 2002. Different aspects of assessing indoor and outdoor thermal comfort. Energy Build. 34, 661-665. https://doi.org/10.1016/S03787788(02)00017-8

Höppe, P., 1999. The physiological equivalent temperature - A universal index for the biometeorological assessment of the thermal environment. Int. J. Biometeorol. 43, 71-75. https://doi.org/10.1007/s004840050118

IBGE, 2013. Censo 2010, Atlas censo demografico. https://doi.org/ISSN 01014234

Iommi, M., Barbera, E., 2015. Thermal Comfort for Older Adults. Cisbat 357362.

ISO. International Organization for Standardization. ISO 10551. Ergonomics of the Thermal Environment - Assessment of the Influence of the Thermal Environment Using Subjective Judgement Scales. Geneva, 1995.

ISO, 2004. ISO 8996 Ergonomics of the thermal environment - Determination of metabolic rate. Management. https://doi.org/10.3403/03205220 
ISO 9920, 2007. Ergonomics of the thermal environment - Estimation of thermal insulation and water vapour resistance of a clothing ensemble. Int. Organ. Stand.

Johansson, E., Thorsson, S., Emmanuel, R., Krüger, E., 2014. Instruments and methods in outdoor thermal comfort studies - The need for standardization. Urban Clim. 10, 346-366. https://doi.org/10.1016/j.uclim.2013.12.002

Lai, D., Guo, D., Hou, Y., Lin, C., Chen, Q., 2014. Studies of outdoor thermal comfort in northern China. Build. Environ. 77, 110-118. https://doi.org/10.1016/j.buildenv.2014.03.026

Larose, J., Boulay, P., Wright-Beatty, H.E., Sigal, R.J., Hardcastle, S., Kenny, G.P., 2014. Age-related differences in heat loss capacity occur under both dry and humid heat stress conditions. J. Appl. Physiol. 117, 69-79. https://doi.org/10.1152/japplphysiol.00123.2014

Lin, T.P., De Dear, R., Hwang, R.L., 2011. Effect of thermal adaptation on seasonal outdoor thermal comfort. Int. J. Climatol. 31, 302-312. https://doi.org/10.1002/joc. 2120

Manu, S., Shukla, Y., Rawal, R., Thomas, L.E., de Dear, R., 2016. Corrigendum to "Field studies of thermal comfort across multiple climate zones for the subcontinent: India model for adaptive comfort (IMAC)" [Building and Environment $98 \quad$ (2016) 5570](S0360132315302171)(10.1016/j.buildenv.2015.12.019). Build. Environ. 106, 422-426. https://doi.org/10.1016/j.buildenv.2016.07.015

Marquardt, D.W., 1970. Generalized Inverses, Ridge Regression, Biased Linear Estimation, and Nonlinear Estimation. Technometrics. https://doi.org/10.2307/1267205

Monteiro, L.M., Alucci, M.P., 2007. Questões teóricas de conforto térmico em espaços abertos: consideração histórica, discussão do estado da arte e proposição de classificação de modelos. Ambient. Construído 7, 43-58.

Monteiro, L.M., Preditivos, M., Térmico, D.E.C., 2008. Modelos Preditivos De Conforto Térmico :

Nasir, R.A., Ahmad, S.S., Ahmed, A.Z., 2012. Psychological Adaptation of Outdoor Thermal Comfort in Shaded Green Spaces in Malaysia. Procedia - Soc. Behav. Sci. 68, 865-878. https://doi.org/10.1016/j.sbspro.2012.12.273

$\mathrm{Ng}$, E., Cheng, V., 2012. Urban human thermal comfort in hot and humid Hong Kong. Energy Build. 55, 51-65. https://doi.org/10.1016/j.enbuild.2011.09.025

Nikolopoulou, M., Baker, N., Steemers, K., 2001. Thermal comfort in outdoor urban spaces: Understanding the Human parameter. Sol. Energy 70, 227-235. https://doi.org/10.1016/S0038-092X(00)00093-1

Okada, M., Kakehashi, M., 2014. The influence of body mass index and outdoor temperature on the autonomic response to eating in healthy young Japanese women. Springerplus 3, 1-10. https://doi.org/10.1186/2193-1801-3-142

Organização Mundial da Saúde, 2015. Relatório Mundial de Envelhecimento e Saúde. Organ. Mund. Saúde 1 , https://doi.org/10.1017/CBO9781107415324.004 
Pantavou, K., Lykoudis, S., Nikolopoulou, M., Tsiros, I.X., 2018. Thermal sensation and climate: a comparison of UTCI and PET thresholds in different climates. Int. J. Biometeorol. 1-14. https://doi.org/10.1007/s00484-018-15694

Pantavou, K., Theoharatos, G., Santamouris, M., Asimakopoulos, D., 2013. Outdoor thermal sensation of pedestrians in a Mediterranean climate and a comparison with UTCI. Build. Environ. 66, 82-95. https://doi.org/10.1016/j.buildenv.2013.02.014

Pickup, J., De Dear, R., 2000. An outdoor thermal comfort index (OUT_SET*)part I-the model and its assumptions., in: In Biometeorology and Urban Climatology at the Turn of the Millenium. Selected Papers from the Conference ICB-ICUC.

Potchter, O., Cohen, P., Lin, T.P., Matzarakis, A., 2018. Outdoor human thermal perception in various climates: A comprehensive review of approaches, methods and quantification. Sci. Total Environ. 631-632, 390-406. https://doi.org/10.1016/j.scitotenv.2018.02.276

Rossi, F.A., Krüger, E.L., Bröde, P., 2012. Definição de faixas de conforto e desconforto térmico para espaços abertos em Curitiba, PR, com o índice UTCI. Ambient. Construído 12, 41-59. https://doi.org/10.1590/S167886212012000100004

Rossi, F.A., Krüger, E.L., Guimarães, I.A., 2013. Modelo preditivo de sensação térmica em espacos abertos em curitiba, pr. RA'E GA - O Espac. Geogr. em Anal. 209-238.

Ruiz, M.A., Correa, E.N., 2015. Adaptive model for outdoor thermal comfort assessment in an Oasis city of arid climate. Build. Environ. 85, 40-51. https://doi.org/10.1016/j.buildenv.2014.11.018

Sartori, M. G. B.; O Vento Norte. Santa Maria: Editora DR Publicidade, 2016. $256 p$.

Schellen, L., van Marken Lichtenbelt, W.D., Loomans, M.G.L.C., Toftum, J., de Wit, M.H., 2010. Differences between young adults and elderly in thermal comfort, productivity, and thermal physiology in response to a moderate temperature drift and a steady-state condition. Indoor Air 20, 273-283. https://doi.org/10.1111/j.1600-0668.2010.00657.x

Spagnolo, J., de Dear, R., 2003. A field study of thermal comfort in outdoor and semi-outdoor environments in subtropical Sydney Australia. Build. Environ. 38, 721-738. https://doi.org/10.1016/S0360-1323(02)00209-3

Tabachnick, B.G., Fidell, L.S., 2012. Using multivariate statistics (6th ed.). New York Harper Row. https://doi.org/10.1037/022267

Thorsson, S., Honjo, T., Lindberg, F., Eliasson, I., Lim, E.M., 2007. Thermal comfort and outdoor activity in Japanese urban public places. Environ. Behav. 39, 660-684. https://doi.org/10.1177/0013916506294937

Trezza, B.M., Apolinario, D., de Oliveira, R.S., Busse, A.L., Gonçalves, F.L.T., Saldiva, P.H.N., Jacob-Filho, W., 2015. Environmental heat exposure and cognitive performance in older adults: a controlled trial. Age (Omaha). 37. https://doi.org/10.1007/s11357-015-9783-z 
United Nations, 2014. World Urbanization Prospects 2014, Demographic Research. https://doi.org/(ST/ESA/SER.A/366)

V. Dubreui; K. P. Fante; O. Planchon; J. L. Sant'Anna Neto., 2018. Climate change evidence in Brazil from Köppen' s climate annual types frequency. Int. J. clim 11, 1-12. https://doi.org/10.1002/joc.5893

Vanos, J.K., Warland, J.S., Gillespie, T.J., Kenny, N.A., 2012. Thermal comfort modelling of body temperature and psychological variations of a human exercising in an outdoor environment. Int. J. Biometeorol. 56, 21-32. https://doi.org/10.1007/s00484-010-0393-2

Villadiego, K., Velay-Dabat, M.A., 2014. Outdoor thermal comfort in a hot and humid climate of Colombia: Afield study in Barranquilla. Build. Environ. 75, 142152. https://doi.org/10.1016/j.buildenv.2014.01.017

WHO, 2017. Guideline: assessing and managing children at primary health-care facilities to prevent overweight and obesity in the context of the double burden of malnutrition Updates for the Integrated Management of Childhood Illness (IMCI), Who.

World Health Organization, 2006. BMI classification. Pharmacotherapy. https://doi.org/10.1001/archinte.1996.00440040101011

Yang, W., Wong, N.H., Jusuf, S.K., 2013. Thermal comfort in outdoor urban spaces in Singapore. Build. Environ. 59, 426-435. https://doi.org/10.1016/j.buildenv.2012.09.008

Zambrano, L., Malafaia, C., Bastos, L., 2006. Thermal comfort evaluation in outdoor space of tropical humid climate. 23rd Conf. Passiv. Low Energy Archit. Geneva, Switz. 6-8. 